Discrete Comput Geom 30:649-669 (2003)

DOI: $10.1007 / \mathrm{s} 00454-003-2713-3$

\title{
The "Point" Goalie Problem
}

\author{
Tom Richardson ${ }^{1}$ and Larry Shepp ${ }^{2}$ \\ ${ }^{1}$ Flarion Technologies, \\ Bedminster, NJ 07921, USA \\ tjr@flarion.com \\ ${ }^{2}$ Statistics Department, Rutgers University, \\ Piscataway, NJ 08854, USA \\ shepp@stat.rutgers.edu
}

\begin{abstract}
Suppose you are given a collection of discs of radius $\varepsilon$ and you are asked to place them in the plane so that any straight line that crosses the unit disc will hit one or more of them. Furthermore, you are asked to do this with as few discs as possible. How many do you need? This problem can be cast as an (infinite) integer programming (IP) problem. Often, for such problems, one can obtain a lower bound by considering the linear programming (LP) relaxation. For the above problem the relaxed problem allows discs with positive weight, requires that each line crossing the unit disc accumulates weight 1 from discs it hits, and asks that the total weight be minimized. This LP problem has a simple asymptotically optimal solution with total weight $1 / \varepsilon$. Clearly, this is the best possible since blocking all the lines in a single direction requires total weight $1 / \varepsilon$.

Although it is quite intuitive that the IP version cannot have such a "perfect" solution, achieving the lower bound, it is surprisingly difficult to obtain a better lower bound. Here we improve the lower bound to $1.001 / \varepsilon$. The true answer is probably considerably larger.

We believe this is the first example of a problem in this class where it has been proven that the LP and IP versions have different answers.
\end{abstract}

\section{Introduction}

By "goalie problems" we mean problems where a goalie (a static set) is required to block every shot (every line passing through) a goal area (another static set). The mathematical problem of interest is to find minimal goalies. The two notions of minimality that arise here are minimal total length and minimal cardinality.

What is the goalie of minimal total length that blocks every shot through the unit disc? In other words, what is the minimal total length (e.g., one-dimensional Hausdorff 


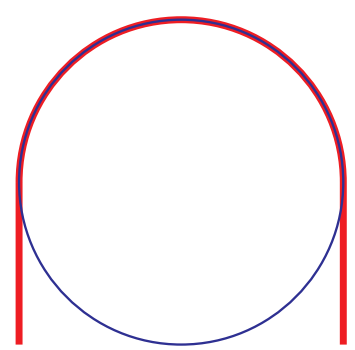

Fig. 1. Shortest connected and shortest rectifiable goalie for the circle.

measure) of a rectifiable set that intersects every line crossing the unit disc? If we require the goalie to be connected, then the optimal solution was proved by Eggleston [Eg] to be the same as the solution under the stronger constraint that the goalie be a rectifiable curve. That problem was solved earlier by Faber et al. [FMP]. The optimal goalie consists of one unit semi-circle and two straight segments each of length 1, see Fig. 1. Thus, the optimal solution has length $\pi+2 \simeq 5.1415926 \ldots$. Faber et al. also found a two piece "goalie" of total length $4.8189 \ldots$. Faber and Mycielski [FM] also indicate a three piece goalie of total length $4.799892 \ldots$. What is the smallest possible total length of a goalie allowing arbitrarily many pieces? To our knowledge the best lower bound is $\pi=3.141 \ldots$, which follows from Crofton's formula [Sa]. Although we believe this lower bound is not tight, we have not been able to prove it. Three-dimensional versions of the problem are discussed in $[\mathrm{Br}]$.

A second form of goalie problem, the focus of this paper, is the following. Given a convex set $C$, how many translates $n$ of $\varepsilon C$ are required to block every line hitting $C$ ? Here the goalie is the set of translates of $\varepsilon C$ and the goal is $C$ itself. We are interested in the asymptotics as $\varepsilon \rightarrow 0$. The trivial lower bound $n \geq \varepsilon^{-1}$ follows by considering one direction. When $C$ is the unit disc we call this problem the point-goalie problem because it is equivalent to asking for the number of points required to ensure that every line hitting the unit disc comes within $\varepsilon$ of one of the points. We use the term $C$-goalie problem to refer to this problem generally.

Both of the above types of goalie problems can be viewed as infinite-dimensional integer programming (IP) problems [KW]. In the first case one considers an infinite collection of tiny line segments and, in the second, the collection of all possible translates of $\varepsilon C$. To each element we attach a variable which takes the value 1 or 0 indicating whether or not that element belongs to the goalie. The cost of the goalie is the sum of all the variables and a linear condition arises from each line hitting the goal: the sum of the variables associated to elements hitting such a line must be at least 1 . If we relax the problem so that the variables may take values in the interval $[0,1]$, then the resulting problem is a linear programming (LP) problem. For example, in the LP version of the point goalie problem we allow the translates of $\varepsilon C$ in the goalie to be weighted and require that the total accumulated weight of a line hitting the blocked set $C$ be at least 1 . In the original IP problem the weight is always 1 (or 0) so a single positive hit suffices. The LP version of the rectifiable set goalie problem allows the set to be weighted: the cost of the goalie is the integral (e.g., with respect to the one-dimensional Hausdorff 
measure) of the weighting function over the goalie and a line accumulates the weight of its points of intersection with the goalie. The accumulated weight for each line hitting the unit disc must be at least 1 . The lower bound $\pi$ on the cost of such a goalie holds for the LP as well as for the IP problem. An optimal solution to the LP problem is obtained by putting weight $\frac{1}{2}$ on the perimeter of the unit disc.

To our knowledge, all lower bounds for goalie problems, except those with connectedness constraints, arise from LP duality. This means that there is no case with a known gap between the optimal solution for the LP version of a goalie problem and the IP version. The main result in this paper is the proof of an LP/IP gap for the point goalie problem ( $C$ is a disc). The magnitude of the established gap is quite small, undoubtedly quite far from the truth.

Perhaps the most studied version of the $C$-goalie problem considers the unit square. Thus, let $S$ be the unit square. The problem is usually cast this way: How many translates of $S$ are required to provide a goalie for $n S$, where $n$ is a positive integer. Bárány and Füredi $[\mathrm{BF}]$ proved the lower bound $\frac{13}{12} n+O(1)$. What if the squares are restricted to lie inside the larger square? L. Fejes Toth [To] conjectured that the answer is $2 n-1$, the solution given by placing the squares along the diagonals. Valtr [Va] found a goalie that uses $1.994 n+O(1)$ squares, disproving Toth's conjecture. The best known lower bound for this problem is $\frac{4}{3} n+O(1)$ which again results from LP duality. This bound was established by Bárány and Füredi $[\mathrm{BF}] .{ }^{1}$ In general, the duals to the $C$-goalie problems consider measures on the space of lines in the plane. The dual measure used to obtain the lower bound of $\frac{4}{3} n$ for $S$ has positive weight on lines in only four directions: those parallel to the sides of the square and those parallel to the diagonals. Thus, the LP version of the square goalie problem has the lower bound $\frac{4}{3} n$ even when the goalie need only block these four directions. We refer to this as the $S_{4 \text { dir }}$ problem. Kern and Wanka [KW] constructed an essentially optimal four direction LP goalie with weight $\frac{4}{3} n+O(1)$ and they also found an IP four direction goalie with weight $\frac{4}{3} n+O(1)$, showing that for the $S_{4 \text { dir }}$ problem the LP and IP optimal values (asymptotically) coincide. Figure 2 shows the LP solution; the IP solution appears as a "rounding" of this one. They also found an all-direction LP goalie of weight $\frac{3}{2} n+O(1)$ and suggested that this might be the best possible. Actually, as we will show in Section 4.2, it turns out that $\frac{4}{3} n$ is (asymptotically) achievable for large $n$ for all directions. The construction of Kern and Wanka [KW] is very close to our solution, the required alterations to their construction become clear in view of an abstraction of the $C$-goalie problem that we call the tomographic goalie problem.

A tomographic goalie for the convex set $C$ is a non-negative function $f$ such that line integrals of $f$ along lines $\ell$ intersecting $C$ are at least $1 / \operatorname{diam}(C, \ell)$, where $\operatorname{diam}(C, \ell)$ is the width of the projection of $C$ parallel to $\ell$. Note that the problem of minimizing $\int f$ is inherently an LP problem. This tomographic goalie problem is asymptotically equivalent to the LP $C$-goalie problem. Requiring the support of $f$ to lie in $C$ is asymptotically equivalent to requiring the translates $\varepsilon C$ comprising a $C$-goalie to intersect $C$.

\footnotetext{
${ }^{1}$ Bárány and Füredi $[\mathrm{BF}]$ also claimed that by considering a dual measure on eight directions they obtained the lower bound $1.43 \cdots n+O(1)$ for the unit square LP problem. In view of the results in Section 4.2 we see that the claim is mistaken.
} 


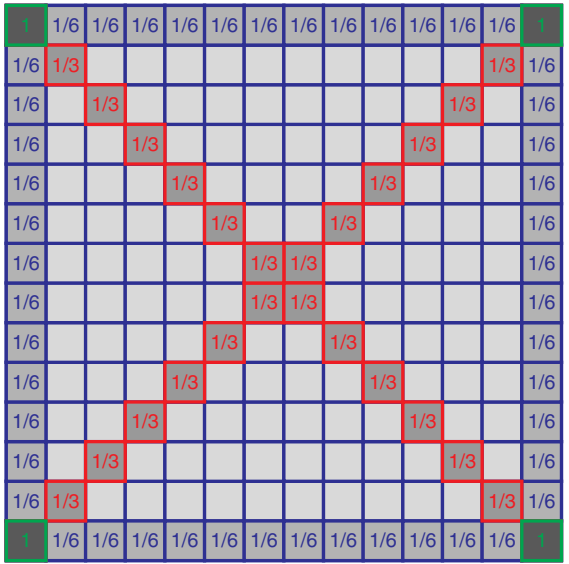

Fig. 2. Kern and Wanka's four direction LP goalie.

\section{Preliminaries}

For concreteness we always consider the convex set $C$ to be closed. It is generally convenient to assume that the origin lies in $C$. A " $C$-goalie" $G$ is a collection of translates of $\varepsilon C$ such that every line that hits $C$ also hits $G$. The parameter $\varepsilon$ is implicit. We use $|G|$ to denote the cardinality of $G$. The lower bound $|G| \geq \varepsilon^{-1}$ follows by considering a single direction.

The LP version of the our problem is formulated as follows. An LP $C$-goalie $G_{\mathrm{LP}}$ is a collection of pairs $\left(x_{i}, w_{i}\right) \in \mathbb{R}^{2} \times \mathbb{R}^{+}, i=1, \ldots, n$, such that for every line $\ell$ intersecting $C$, we have

$$
\sum\left\{w_{i}:\left\{\varepsilon C+x_{i}\right\} \cap \ell \neq \emptyset\right\} \geq 1 .
$$

In this case we define $\left|G_{\mathrm{LP}}\right|:=\sum_{i=1}^{n} w_{i}$ and the lower bound $\left|G_{\mathrm{LP}}\right| \geq \varepsilon^{-1}$ again follows by considering a single direction. Note that an LP $C$-goalie is a $C$-goalie when we constrain $w_{i} \in\{0,1\}$. Let $L P_{\varepsilon}(C)$ denote the infimum of $\varepsilon\left|G_{\mathrm{LP}}\right|$ over all LP $C$-goalies and define

$$
L P(C):=\limsup _{\varepsilon \rightarrow 0} L P_{\varepsilon}(C)
$$

\subsection{Asymptotic Equivalence to the Tomographic Goalie Problem}

Consider now the class $\Phi(C) \subset L_{1}$ of non-negative (measurable) functions $f$ such that, whenever $\ell \cap C \neq \emptyset$, we have

$$
\operatorname{diam}(C, \ell) \int_{\ell} f \geq 1
$$


where $\int_{\ell} f$ denotes the line integral of $f$ along $\ell$. We refer to such an $f$ as a tomographic goalie for $C$. Let us define the following constant:

$$
T G(C):=\inf _{f \in \Phi(C)} \int_{\mathbb{R}^{2}} f(x) d x .
$$

The trivial lower bound $T G(C) \geq 1$ is known to be tight if and only if $C$ is the affine image of the disc, i.e., an ellipse [MS]. That is, if $C$ supports a function $f$ (see the function $f=\xi$ below) whose marginal in every direction is a boxcar step function (constant on the projection of $C$ and zero outside the projection of $C$ ), then $C$ is an ellipse.

Other quantities of interest may be defined by restricting goalies to be supported in $C$. Thus, we define $L P_{\text {in }}(C)$ and $T G_{\text {in }}(C)$ corresponding to $L P(C)$ and $T G(C)$ with this extra condition imposed. (For $L P_{\text {in }}$ the condition is that translates of $\varepsilon C$ should have non-empty intersection with $C$.)

In the case of the unit disc $D$, the following function provides an optimal tomographic goalie:

$$
\xi(y):= \begin{cases}\frac{1}{2 \pi} \frac{1}{\sqrt{1-\|y\|^{2}}}, & \|y\|<1, \\ 0, & \|y\| \geq 0 .\end{cases}
$$

Line integrals of $\xi$ are $\frac{1}{2}$ or 0 according to whether the distance of the line from the origin is less than 1 or not, i.e., $\xi$ is a tomographic goalie for the unit disc $D$. By construction, we now have

$$
T G(D)=T G_{\text {in }}(D)=1 .
$$

By discretizing $\xi$ on a scale much smaller than $\varepsilon$, one can construct a weak $\varepsilon$ point goalie whose total weight is only slightly larger than $\int \xi(x) d x$. The idea is illustrated in Fig. 3.
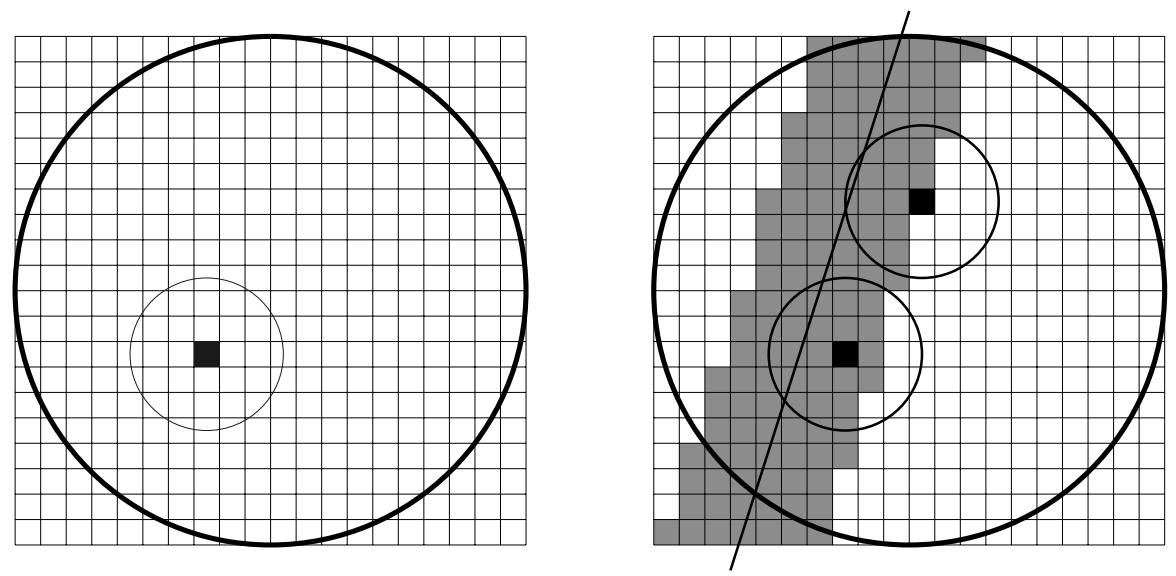

Fig. 3. Making an LP point goalie from a tomographic goalie: Tile the plane with squares of side length $\varepsilon^{2}$. For each square, center a circle of radius $\varepsilon$ on the square. Set the weight equal to the $\varepsilon^{-1}$ times the integral of $\xi$ over the square. A line intersecting the unit disc accumulates total weight approximately equal to the integral of $\varepsilon^{-1} \xi$ over a parallel swath of width $2 \varepsilon$, which is approximately 1 . 
We can use this approach more generally for any bounded convex set $C$ to conclude

$$
T G(C) \leq L P(C) \quad \text { and } \quad T G_{\text {in }}(C) \leq L P_{\text {in }}(C) .
$$

We now consider the converse: constructing a tomographic goalie from an LP $C$ goalie. Assume that the origin is contained in the interior of $C$. Given an LP $C$-goalie consider the measure obtained by placing a point mass of weight $w$ at $x$ whenever $x+\varepsilon C$ is an element of the goalie with weight $w$. Consider now the function obtained by taking for each point $x$ the total mass of the measure contained in a disc of radius $\varepsilon^{1 / 2}$ centered at $x$ divided by the area of the disc $\pi \varepsilon$. It is not hard to see that, for small $\varepsilon$, the resulting function is nearly a tomographic goalie for $C$ and that, with small adjustments, it can be made into tomographic goalie for $C$. Taking limits as $\varepsilon$ tends to zero we obtain

$$
T G(C) \geq L P(C) \quad \text { and } \quad T G_{\text {in }}(C) \geq L P_{\text {in }}(C),
$$

which, together with (1), establishes the asymptotic equivalence of the tomographic goalie problem for $C$ and the LP $C$-goalie problem.

In general the tomographic goalie problem for $C$ is easier to study than the $C$-goalie problem. In Section 4 we present some optimal tomographic goalies for the triangle and the square.

\subsection{Lines in the Plane}

We parameterize lines in the plane as follows (see Fig. 4). Fix, e.g., the $x$-axis. Let $\theta \in[0, \pi)$ and let $p \in \mathbb{R}$. Let $\vec{\theta}$ denote the unit vector making angle $\theta$ with the $x$-axis. The line $\ell(\theta, p)$ is the line with normal $\vec{\theta}$ that contains the point $p \vec{\theta}$. Note that $p \vec{\theta}$ is the closest point in $\ell(\theta, p)$ to the origin. Let $d \mu=d \theta d p$ be the so-called kinematic density on lines $\ell(\theta, p)$ in $\mathbb{R}^{2}$ normalized so that $\mu[D]=2 \pi$ where, for any set $A \subset \mathbb{R}^{2}$, we let $[A]$ denote the set of lines hitting $A$. For any convex set $C$, Crofton's formula states

$$
\mu[C]=H_{1}(\partial C),
$$

where $H_{1}(\partial C)$ denotes the total length of the boundary of $C$.

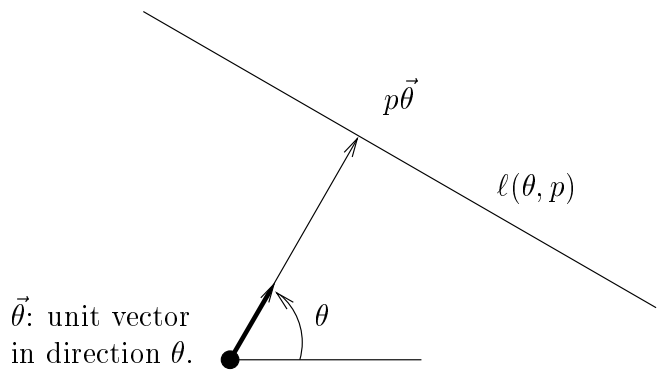

Fig. 4. Representation of lines in the plane. 


\section{An LP/IP Gap for the Unit Disc}

In this section we establish the existence of an LP/IP gap for the $D$-goalie problem. Since we focus exclusively on the unit disc, we suppress $D$ from the notation and refer simply to the goalie problem. We generally denote discs by $B$, sometimes $B_{x}$ to indicate the center $x$. The radius is $\varepsilon$ unless stated otherwise. Given distinct points $x$ and $y$ let $\overline{x y}$ denote the line through $x$ and $y$.

The key idea of the proof is to use $\mu$ and argue that with a specific number of discs one can cover only an insufficient $\mu$-fraction of the lines through $D$. Henceforth, let $\delta$ denote the constant 0.001 . We will prove the following.

Theorem 1. For any $\varepsilon<1$, a goalie $G$ must satisfy $\varepsilon|G| \geq 1+\delta$.

One might suspect that the rigidity of the "perfect goalie" condition gives rise to Theorem 1. This is not the case: we can relax the condition that $G$ be a perfect goalie without sacrificing the conclusion. (See Theorem 2.)

For notational simplicity let $\mu_{D}$ denote the restriction of $\mu$ to $[D]$, i.e., $\mu_{D}(A)=$ $\mu([D] \cap A)$. We say that $G$ is an $\eta$-almost goalie if

$$
\mu_{D}([G]) \geq(1-\eta) 2 \pi,
$$

where

$$
[G]:=\bigcup_{x_{i} \in G}\left[B_{x_{i}}\right] .
$$

We prove Theorem 1 by proving the following stronger result. (We assume throughout that $\varepsilon<1-\delta$.)

Theorem 2. Any $\delta$-almost goalie $G$ satisfies $\varepsilon|G| \geq 1+\delta$.

Before presenting the proof of Theorem 2 we briefly sketch the main ideas. Imagine constructing the goalie by placing one $\varepsilon$-disc at a time. Each new disc placed covers some measure of lines not already covered by previous discs. Without loss of generality we can assume that this quantity is decreasing. If the goalie is nearly optimal, i.e., close to the lower bound, then most discs must be effective in the sense that the measure of additional lines covered by the disc must be near the maximum possible. We can afford to have only an asymptotically negligible number of ineffective discs. Let us remove them from consideration. All of the effective discs must be well separated from each other since a close pair would share a significant fraction of the lines they cover. Now, consider expanding an effective disc by a factor $\alpha>1$. Lines through the center core of the expanded disc are covered by the original disc. Lines which pass through the expanded disc but not through the original disc can only be covered by other discs in the goalie and most of these lines will indeed typically need to be covered by other, relatively distant, effective discs. Because this coverage is accomplished by distant discs, however, a significant fraction of the lines passing through the original effective disc must also be covered by those distant effective discs and we thereby obtain a contradiction.

We now turn to the proof of Theorem 2. 


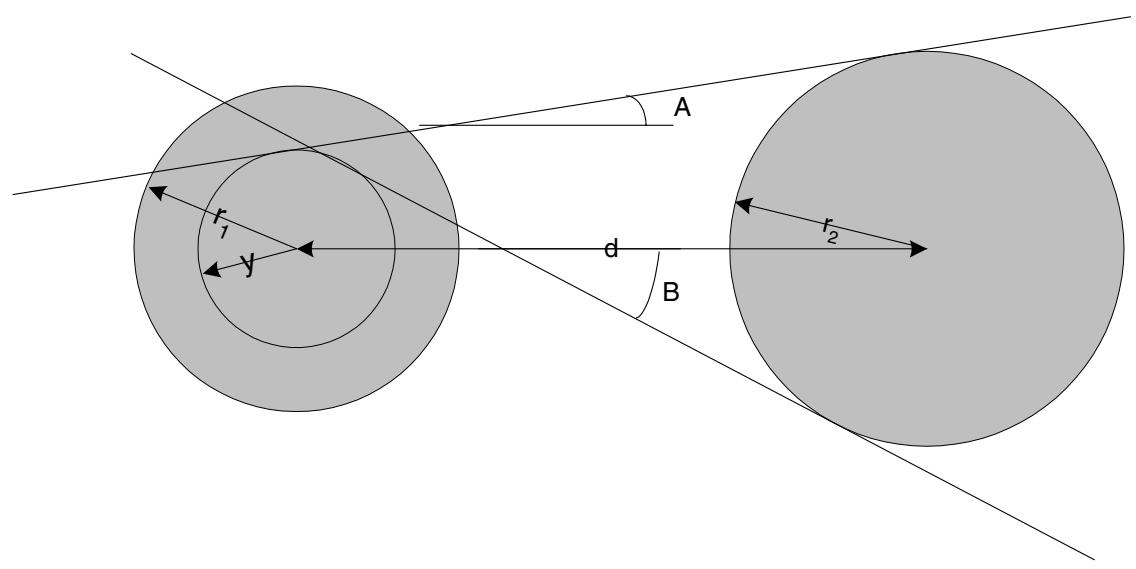

Fig. 5. Illustration of Lemma 1. Angle $A$ is $\sin ^{-1}\left(\left(r_{2}-y\right) / d\right)$ and angle $B$ is $\sin ^{-1}\left(\left(r_{2}+y\right) / d\right)$.

In order to distinguish IP from LP we need two discs to interact: the following elementary inequality is fundamental.

Lemma 1. Let $B_{x_{1}}$ and $B_{x_{2}}$ be two discs of radius $r_{1}$ and $r_{2} \geq r_{1}$ respectively and let $d:=\left\|x_{1}-x_{2}\right\|$. Then $\mu\left(\left[B_{x_{1}}\right] \cap\left[B_{x_{2}}\right)\right]$ is decreasing in $d$ and for $d \geq r_{1}+r_{2}$ we have

$$
\frac{4 r_{1} r_{2}}{d} \leq \mu\left(\left[B_{x_{1}}\right] \cap\left[B_{x_{2}}\right]\right) \leq 2 r_{1} \sin ^{-1}\left(\frac{2 r_{2}}{d}\right) .
$$

Proof. That $\mu\left(\left[B_{x_{1}}\right] \cap\left[B_{x_{2}}\right]\right)$ is decreasing in $d$ is clear: hold $x_{1}$ fixed and consider moving $x_{2}$ along a line through $x_{1}$, then $\left[B_{x_{1}}\right] \cap\left[B_{x_{2}}\right]$ is decreasing in $d$.

Assume $d \geq r_{1}+r_{2}$. Elementary geometry, see Fig. 5, shows that the set of lines intersecting $B_{x_{1}}$ at distance $y \in\left[0, r_{1}\right]$ from $x_{1}$ that also intersect $B_{x_{2}}$ are precisely those that cross $\overline{x_{1} x_{2}}$ at an angle in the range $\left(\sin ^{-1}\left(\left(y-r_{2}\right) / d\right), \sin ^{-1}\left(\left(y+r_{2}\right) / d\right)\right)$. Hence, we obtain

$$
\begin{aligned}
\mu\left(\left[B_{x_{1}}\right] \cap\left[B_{x_{2}}\right]\right) & =\int_{-r_{1}}^{r_{1}} \sin ^{-1}\left(\frac{y+r_{2}}{d}\right)-\sin ^{-1}\left(\frac{y-r_{2}}{d}\right) d y \\
& =\int_{-r_{1}}^{r_{1}}\left(\int_{\left(y-r_{2}\right) / d}^{\left(y+r_{2}\right) / d} \frac{1}{\sqrt{1-u^{2}}} d u\right) d y
\end{aligned}
$$

The inequality $\left(4 r_{1} r_{2}\right) / d \leq \mu\left(\left[B_{x_{1}}\right] \cap\left[B_{x_{2}}\right]\right)$ follows easily since $\left(1 / \sqrt{1-u^{2}} \geq 1\right.$. Now, note that for $y \in\left(-r_{1}, r_{1}\right)$ we have

$$
\int_{\left(y-r_{2}\right) / d}^{\left(y+r_{2}\right) / d} \frac{1}{\sqrt{1-u^{2}}} d u \leq \int_{0}^{2 r_{2} / d} \frac{1}{\sqrt{1-u^{2}}} d u=\sin ^{-1}\left(\frac{2 r_{2}}{d}\right)
$$

and the right inequality follows. 
We shall also require the following elementary result.

Lemma 2. Let $B_{x}$ be a disc of radius $\varepsilon$ disjoint from $D$. Then

$$
\mu([B] \backslash[D]) \geq 4 \varepsilon \cos ^{-1} \frac{1+\varepsilon}{\|x\|} .
$$

Proof. Assume $\|x\| \geq 1+\varepsilon$. Let $\psi=\cos ^{-1}((1+\varepsilon) /\|x\|)$. Any line that crosses $\overline{0 x}$ at an angle in $[\pi / 2-\psi, \pi / 2+\psi]$ and intersects $B$ does not intersect $D$. It follows that $\mu([B] \backslash[D]) \geq 4 \varepsilon \psi$.

Assume that $G$ is a $\delta$-almost goalie satisfying $\varepsilon|G| \leq 1+\delta$. We then have the following inequality:

$$
\sum_{B \in G} \mu([B])-\mu_{D}([G]) \leq 4 \pi \delta .
$$

This follows since $|G| \leq(1+\delta) / \varepsilon, \mu([B])=2 \pi \varepsilon$ for each disc $B \in G$, and $\mu_{D}([G]) \geq$ $(1-\delta) 2 \pi$. This later inequality also implies

$$
|G| \geq \frac{1-\delta}{\varepsilon}
$$

As a first step in the proof we eliminate the possibility of solutions for relatively large $\varepsilon$.

Lemma 3. If $\varepsilon \in(0.01,1-\delta)$, then any $\delta$-almost goalie $G$ satisfies $\varepsilon|G|>1+\delta$.

Proof. Assume that $\varepsilon \in(0.01,1-\delta)$ and that $G$ is a $\delta$-almost goalie satisfying $\varepsilon|G| \leq$ $1+\delta$. Define

$$
R:=\max _{B_{x} \in G}\|x\|
$$

Equation (2) implies that for any $B_{x} \in G$ we have $4 \pi \delta \geq \mu\left(\left[B_{x}\right] \backslash[D]\right)$. If $\|x\| \geq 1+\varepsilon$, then Lemma 2 provides $\mu\left(\left[B_{x}\right] \backslash[D]\right) \geq 4 \varepsilon \cos ^{-1}((1+\varepsilon) /\|x\|)$ which now yields $\|x\| \leq$ $(1+\varepsilon) / \cos (\pi \delta / \varepsilon)$. Since $\varepsilon \geq 0.01$ we easily obtain

$$
R \leq 1.06(1+\varepsilon)
$$

Clearly, $|G| \geq 2$. Let $B_{x_{1}}$ and $B_{x_{2}}$ be two discs in $G$ and let $d:=\left\|x_{1}-x_{2}\right\|$. By (2) we have $\mu\left(\left[B_{x_{1}}\right] \cap\left[B_{x_{2}}\right]\right) \leq 4 \pi \delta$. Since $4 \pi \delta<2 \varepsilon$ we conclude from Lemma 1 that all discs in $G$ are disjoint and, noting $d \leq 2 R$, that

$$
\frac{2 \varepsilon^{2}}{R} \leq \frac{4 \varepsilon^{2}}{d} \leq \mu\left(\left[B_{x_{1}}\right] \cap\left[B_{x_{2}}\right]\right)
$$


Now, we order the discs in $G$ arbitrarily $B_{1}, \ldots, B_{|G|}$ and write

$$
\begin{aligned}
(|G|-1) \frac{2 \varepsilon^{2}}{R} & \leq \sum_{i=2}^{|G|} \mu\left(\left[B_{i}\right] \cap\left[B_{i-1}\right]\right) \\
& \leq \sum_{i=2}^{|G|} \mu\left(\left[B_{i}\right] \cap\left[\bigcup_{j=1}^{i-1} B_{j}\right]\right) \\
& =\sum_{i=1}^{|G|} \mu\left(\left[B_{i}\right]\right)-\mu([G]) \\
& \leq \sum_{i=1}^{|G|} \mu\left(\left[B_{i}\right]\right)-\mu_{D}([G]) \\
& \leq 4 \pi \delta,
\end{aligned}
$$

where the last step follows from (2). Recalling $|G| \geq(1-\delta) / \varepsilon$ and $R \leq 1.06(1+\varepsilon)$, we have

$$
(1-\delta-\varepsilon) \varepsilon \leq 2 \pi \delta(1.06(1+\varepsilon))
$$

from which we easily conclude $\varepsilon \leq 0.01$.

Assume $G:=\left\{B_{i}\right\}_{i=1, \ldots, n}$ is a $\delta$-almost goalie satisfying $\varepsilon|G| \leq 1+\delta$, where $\varepsilon<1-\delta$. We now know that $\varepsilon \leq 0.01$.

Consider placing the discs in $G$ one at a time. Define $S_{0}:=\emptyset$ and for $i=1, \ldots, n$ let $S_{i}:=\bigcup_{j=1}^{i} B_{j}$. We can write $[G]$ as a disjoint union as follows:

$$
[G]=\bigcup_{i=1}^{n}\left[B_{i}\right] \backslash\left[S_{i-1}\right] .
$$

The quantity $\mu\left(\left[B_{i}\right] \backslash\left[S_{i-1}\right]\right)$ measures the effectiveness of adding disc $B_{i}$ to the collection $S_{i-1}$. (Note that we might have used $\mu_{D}$ here instead of $\mu$ but this definition is slightly more convenient.) We may assume that the discs $B_{i}$ are ordered with decreasing effectiveness. Indeed, let $B_{n}$ maximize $\mu\left(\left[B_{n}\right] \cap\left[G \backslash B_{n}\right]\right) ; B_{n}$ is the least effective disc in $G$. Given $B_{i+1}, \ldots, B_{n}$ we have $S_{i}=G \backslash \bigcup_{j=i+1}^{n} B_{j}$. Now, let $B_{i} \in S_{i}$ maximize $\mu\left(\left[B_{i}\right] \cap\left[S_{i} \backslash B_{i}\right]\right)$. It follows easily that the effectiveness $\mu\left(\left[B_{i}\right] \backslash\left[S_{i-1}\right]\right)$ is decreasing, or, equivalently, that the waste $\mu\left(\left[B_{i}\right] \cap\left[S_{i-1}\right]\right)$ is increasing in $i$. Moreover, for each disc $B \in S_{i}$ we have $\mu\left([B] \cap\left[S_{i} \backslash B\right]\right) \leq \mu\left(\left[B_{i}\right] \cap\left[S_{i} \backslash B_{i}\right]\right)$.

We now establish a relationship between the waste and the coverage after the ith step. Define the normalized waste

$$
W_{i}:=\frac{\mu\left(\left[B_{i}\right] \cap\left[S_{i-1}\right]\right)}{2 \pi \varepsilon}
$$

which is increasing $i$.

Lemma 4. For each $i=1, \ldots, n-1$ we have

$$
\mu_{D}\left(\left[S_{i}\right]\right) \geq 2 \pi\left(1-2 \delta W_{i+1}^{-1}\right) .
$$


Proof. We first note that

$$
\begin{aligned}
\mu([G]) & =\mu\left(\left[S_{i}\right]\right)+\sum_{j=i+1}^{n} \mu\left(\left[B_{j}\right]\right)\left(1-W_{j}\right) \\
& \leq \mu\left(\left[S_{i}\right]\right)+\sum_{j=i+1}^{n} \mu\left(\left[B_{j}\right]\right)\left(1-W_{i+1}\right) .
\end{aligned}
$$

From this we obtain

$$
\begin{aligned}
W_{i+1} \sum_{j=1}^{n} \mu\left(\left[B_{j}\right]\right) & \leq \mu\left(\left[S_{i}\right]\right)+\sum_{j=i+1}^{n} \mu\left(\left[B_{j}\right]\right)-\mu([G]) \\
& \leq \sum_{j=1}^{n} \mu\left(\left[B_{j}\right]\right)-\mu([G]) \\
& \leq \sum_{j=1}^{n} \mu\left(\left[B_{j}\right]\right)-\mu_{D}([G]) \\
& \leq 4 \pi \delta
\end{aligned}
$$

where for the last step we applied (2). Substituting this into (4) and noting that $\mu([G])-$ $\mu_{D}([G]) \geq \mu([S])-\mu_{D}([S])$ we obtain

$$
\begin{aligned}
\mu_{D}\left(\left[S_{i}\right]\right) & \geq \mu_{D}([G])-\sum_{j=i+1}^{n} \mu\left(\left[B_{j}\right]\right)\left(1-W_{i+1}\right) \\
& \geq(1-\delta) 2 \pi-\frac{4 \pi \delta}{W_{i+1}}\left(1-W_{i+1}\right) \\
& =2 \pi\left(1-2 \delta W_{i+1}^{-1}\right) .
\end{aligned}
$$

For convenience and definiteness we set $W=10 \delta$ and choose $i$ so that $W_{i} \leq W \leq$ $W_{i+1}$. (If $W_{n} \leq W$, then choose $i=n$.) Henceforth we let $S$ denote $S_{i}$. From the above lemma we now have

$$
\mu_{D}([S]) \geq 1.6 \pi
$$

The remainder of the proof focuses on $S$, that is, $G$ pruned of the $n-i$ least effective discs.

Let $B_{x_{0}}$ be any disc in $S$ and consider re-ordering the discs in $S$ according to distance from $B_{x_{0}}$. Thus, $S=\bigcup_{i=0}^{|S|-1} B_{x_{i}}$ where $\left\|x_{i}-x_{0}\right\|$ is non-decreasing in $i$. We abbreviate $B_{x_{i}}$ as $B_{i}$. We now partition $S \backslash B_{0}$ into subsets $T_{j}$, we define the sets $T_{j}$ recursively. Let $R_{1}=S \backslash B_{0}=\left\{B_{1}, \ldots, B_{|S|-1}\right\}$. Given $R_{j} \subset S, R_{j} \neq \emptyset$, let $i_{j}$ be the smallest index among discs in $R_{j}$ and set

$$
T_{j}:=\left\{B \in R_{j}: \mu\left([B] \cap\left[B_{i_{j}}\right] \cap\left[B_{0}\right]\right) \neq 0\right\} .
$$

(Note that $B_{i_{j}} \in T_{j}$.) We then define

$$
R_{j+1}:=R_{j} \backslash T_{j}
$$


If $R_{m+1}=\emptyset$, which holds for some $m$ large enough, then $T_{j}, j=1, \ldots, m$, is the desired partition. Note that whenever $1 \leq j<k \leq m$ we have

$$
\mu\left(\left[B_{i_{j}}\right] \cap\left[B_{i_{k}}\right] \cap\left[B_{0}\right]\right)=0 .
$$

The main point of this is that it enables us to write

$$
\sum_{j} \mu\left(\left[B_{0}\right] \cap\left[B_{i_{j}}\right]\right)=\mu\left(\left[B_{0}\right] \cap\left[\bigcup_{j=1}^{m} B_{i_{j}}\right]\right)
$$

and since

$$
\mu\left(\left[B_{0}\right] \cap\left[\bigcup_{j=1}^{m} B_{i_{j}}\right]\right) \leq \mu\left(\left[B_{0}\right] \cap\left[S \backslash B_{0}\right]\right) \leq W 2 \pi \varepsilon
$$

we obtain

$$
\sum_{j} \mu\left(\left[B_{0}\right] \cap\left[B_{i_{j}}\right]\right) \leq W 2 \pi \varepsilon .
$$

Our goal now is to bound $\sum_{j} \mu\left(\left[B_{0}\right] \cap\left[B_{i_{j}}\right]\right)$ from below for some particular disc $B_{0}$. We consider expanding the discs in $S$ by a factor $\alpha$. For a given disc $B, \alpha B$ denotes the disc with the same center as $B$ but linearly scaled by $\alpha$, i.e., the radius of $\alpha B$ is $\alpha$ times that of $B$. We want to show that on average $\mu\left(\left[\alpha B_{0}\right] \cap\left[S \backslash B_{0}\right]\right)$ is fairly large, nearly equal to $\mu\left(\left[\alpha B_{0}\right] \backslash\left[B_{0}\right]\right)$. We then proceed through a series of inequalities as follows:

$$
\begin{aligned}
C_{1} & \leq \mu\left(\left[S \backslash B_{0}\right] \cap\left[\alpha B_{0}\right]\right) \\
& \leq \sum_{j} \mu\left(\left[T_{j}\right] \cap\left[\alpha B_{0}\right]\right) \\
& \leq \sum_{j} \mu\left(\left[r B_{i_{j}}\right] \cap\left[\alpha B_{0}\right]\right) \\
& \leq C_{2} \sum_{j} \mu\left(\left[B_{i_{j}}\right] \cap\left[B_{0}\right]\right),
\end{aligned}
$$

where $C_{1}, C_{2}$, and $r$ are positive constants, producing a contradiction to (6). Inequality (8) is obvious since $S \backslash B_{0}=\bigcup_{j} T_{j}$. Inequalities (9) and (10) are relatively straightforward to prove and do not depend on the choice of $B_{0}$. Therefore, we prove these two steps first.

Lemma 5. For each $j$ and any $\alpha \geq 1$ we have

$$
\left[T_{j}\right] \cap\left[\alpha B_{0}\right] \subset\left[(5+2 \alpha) B_{i_{j}}\right] \cap\left[\alpha B_{0}\right] .
$$

Proof. It is clear that for sufficiently large $r$ we have

$$
\left[T_{j}\right] \cap\left[\alpha B_{0}\right] \subset\left[r B_{i_{j}}\right] \cap\left[\alpha B_{0}\right] .
$$

Elementary considerations, see Fig. 6 , reveal that $r=\alpha \varepsilon+d \sin (2 A+B)$ where $A=\sin ^{-1}(2 \varepsilon / d)$ and $B=\sin ^{-1}(((\alpha+1) \varepsilon) / d)$ is precisely the required value. The inequality $\sin (2 A+B) \leq 2 \sin A+\sin B$ now yields the desired result. 


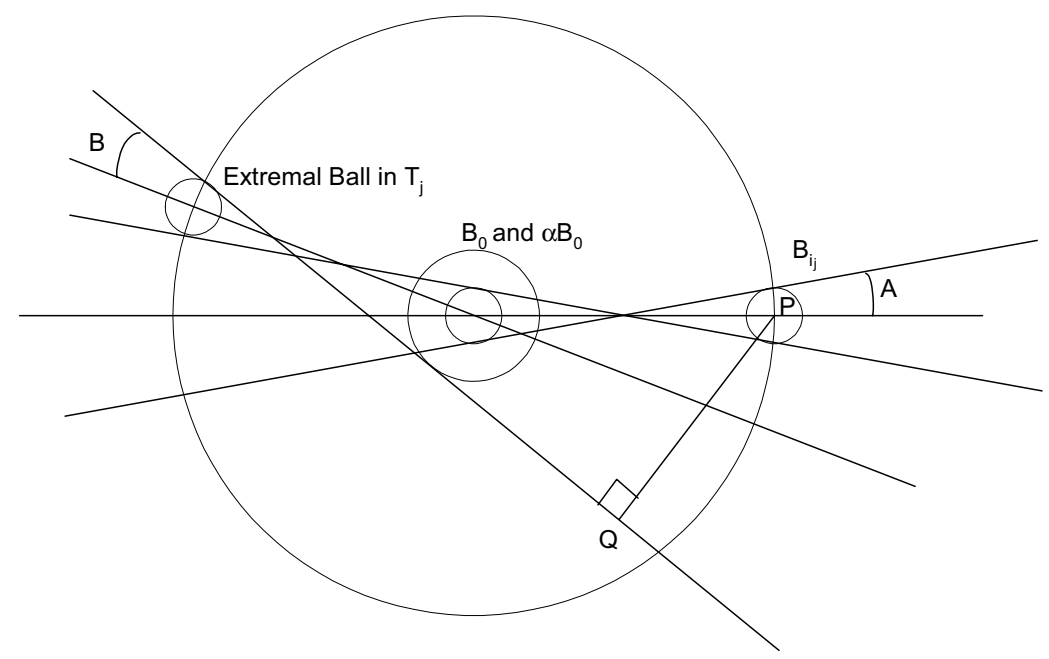

Fig. 6. The small discs $B_{0}$ and $B_{i_{j}}$ have radius $\varepsilon$. An extremal disc from $T_{j}$ is depicted. Also shown is a disc of radius $\alpha \varepsilon$ and a disc of radius $d$, both centered at the origin. The line segment PQ is seen to have length $\alpha \varepsilon+\sin ^{-1}(2 A+B)$ while $A=\sin ^{-1}(2 \varepsilon / d)$ and $B=\sin ^{-1}((\alpha+1) \varepsilon / d)$.

Lemma 5 is clearly sufficient to prove (9) with $\alpha=3$ and $r=5+2 \alpha=11$. We now consider (10).

Lemma 6. The centers of any pair of discs in $S$ are separated by a distance of at least $(2 / W \pi) \varepsilon$.

Proof. Let $B_{1}$ and $B_{2}$ be two discs from $S$. We have $\mu\left(\left[B_{1}\right] \cap\left[B_{2}\right]\right) \leq W 2 \pi \varepsilon$. Denote the distance between the centers of the discs by $d$. Applying Lemma 1 we obtain $W 2 \pi \varepsilon \geq$ $4 \varepsilon^{2} / d$ which yields the desired result.

Let us now assume that $\alpha=3$. Lemma 6 now implies that $(5+2 \alpha) B_{i_{j}}$ and $\alpha B_{0}$ are disjoint so we can apply Lemma 1 to write

$$
\mu\left(\left[(5+2 \alpha) B_{i_{j}}\right] \cap\left[\alpha B_{0}\right]\right) \leq \alpha \frac{\sin ^{-1}((5+2 \alpha)(2 \varepsilon / d))}{(2 \varepsilon / d)} \mu\left(\left[B_{i_{j}}\right] \cap\left[B_{0}\right]\right),
$$

where $d$ is the distance between the centers of $B_{0}$ and $B_{i_{j}}$. Applying Lemma 6 again we have $2 \varepsilon / d \leq W \pi$ from which we easily conclude $\left(\sin ^{-1}(5+2 \alpha)(2 \varepsilon / d)\right) /(2 \varepsilon / d) \leq 12$. Thus, we have now established (10) with $C_{2}=36$, assuming $\alpha=3$.

Finally we turn to the proof of (7). We now consider expanding all of the discs in $S$ by a factor of $\alpha=3$. We are interested in knowing how well the lines through the expanded discs are covered by $S$. Note that the projection of the expanded set, counting multiplicities, equals the sum of the projection of $S$ and two translates of that projection. 
Since the projection $S$ equals 1 on most lines through $D$, the expanded projection will equal 3 on most lines through $D$ and the two projections will have a large correlation. This is the key observation underlying the following result.

Lemma 7. Let $\alpha=3$, then

$$
\sum_{B \in S} \mu_{D}([S \backslash B] \cap[\alpha B]) \geq 5 \mu_{D}([S])-(4+4 \varepsilon) \pi-\sum_{B \in S} \mu_{D}([B]) .
$$

Proof. First, note that for each $B \in S$, we have

$$
\begin{aligned}
\mu_{D}([S \backslash B] \cap[\alpha B]) & \geq \mu_{D}([S] \cap[\alpha B])-\mu_{D}([B] \cap[\alpha B]) \\
& =\mu_{D}([S] \cap[\alpha B])-\mu_{D}([B])
\end{aligned}
$$

and we see that (11) is implied by

$$
\sum_{B \in S} \mu_{D}([S] \cap[3 B]) \geq 5 \mu_{D}([S])-(4+4 \varepsilon) \pi .
$$

Let $f_{\theta}^{S}$ be the projection (an indicator function) of $S$ in the direction $\theta$ and, similarly, let $f_{\theta}^{B}$ be the projection of the disc $B$. Introduce an $(x$-)axis perpendicular to the $\theta$ direction and, for simplicity, assume that the origin projects to 0 on that axis.

Let $B$ be any disc in $S$. Then it is easy to see that

$$
\mu_{D}([S] \cap[B])=\int_{0}^{\pi} \int_{-1}^{1} f_{\theta}^{S}(x) f_{\theta}^{B}(x) d x d \theta
$$

and that

$$
\begin{aligned}
\mu_{D}([S] \cap[3 B]) & =\int_{0}^{\pi} \int_{-1}^{1} f_{\theta}^{S}(x) f_{\theta}^{3 B}(x) d x d \theta \\
& =\int_{0}^{\pi} \int_{-1}^{1} f_{\theta}^{S}(x)\left(f_{\theta}^{B}(x+2 \varepsilon)+f_{\theta}^{B}(x)+f_{\theta}^{B}(x-2 \varepsilon)\right) d x d \theta .
\end{aligned}
$$

Since $\sum_{B \in S} f_{\theta}^{B} \geq f_{\theta}^{S}$, we now have

$$
\sum_{B \in S} \mu_{D}([S] \cap[3 B]) \geq \int_{0}^{\pi} \int_{-1}^{1} f_{\theta}^{S}(x)\left(f_{\theta}^{S}(x+2 \varepsilon)+f_{\theta}^{S}(x)+f_{\theta}^{S}(x-2 \varepsilon)\right) d x d \theta .
$$

Now, since $f_{\theta}^{S}$ takes only the values 0 and 1 we have $\int_{-1}^{1} f_{\theta}^{S}(x-t) d x \geq \int_{-1}^{1} f_{\theta}^{S}(x)-|t|$ and $\left(1-f_{\theta}^{S}(x)\right)\left(1-f_{\theta}^{S}(x-t)\right) \geq 0$. Thus,

$$
\begin{aligned}
\int_{-1}^{1} f_{\theta}^{S}(x) f_{\theta}^{S}(x-t) d x & \geq \int_{-1}^{1}\left(f_{\theta}^{S}(x)+f_{\theta}^{S}(x-t)-1\right) d x \\
& \geq 2 \int_{-1}^{1} f_{\theta}^{S}(x) d x-|t|-2 .
\end{aligned}
$$


By integrating with respect to $\theta$, (14) now yields

$$
\int_{0}^{\pi} \int_{-1}^{1} f_{\theta}^{S}(x) f_{\theta}^{S}(x-t) d x d \theta \geq 2 \mu_{D}([S])-(2+|t|) \pi .
$$

Of course, in the case $t=0$ we have the slightly stronger result $\int_{0}^{\pi} \int_{-1}^{1} f_{\theta}^{S}(x) f_{\theta}^{S}(x)$ $d x d \theta=\mu_{D}([S])$. Thus, from (13) we now obtain (12).

Since $(1 /|S|) \sum_{B \in S} \mu_{D}([B]) \leq 2 \pi \varepsilon$ and $|S| \leq|G| \leq(1+\delta) / \varepsilon$, we now see that there exists a disc $B_{0} \in S$ such that

$$
\begin{aligned}
\mu\left(\left[S \backslash B_{0}\right] \cap\left[3 B_{0}\right]\right) & \geq \frac{1}{|S|}\left(5 \mu_{D}([S])-(4+4 \varepsilon) \pi\right)-2 \pi \varepsilon \\
& \geq \frac{\varepsilon}{1+\delta}(8 \pi-4-4 \varepsilon)-2 \pi \varepsilon \\
& \geq 14 \varepsilon,
\end{aligned}
$$

where for the second inequality we have applied (5). Thus, we have now established (7) with $\alpha=3$ and $C_{1}=14 \varepsilon$.

We have now proved (7)-(10) with $C_{1}=14 \varepsilon$ and $C_{2}=36$. Substituting from (6) we now obtain

$$
\begin{aligned}
14 \varepsilon & \leq 36 \sum_{j} \mu\left(\left[B_{0}\right] \cap\left[B_{i_{j}}\right]\right) \\
& \leq 36 W 2 \pi \varepsilon \\
& \leq 3 \varepsilon
\end{aligned}
$$

which is a contradiction. Thus, the proof of Theorem 2 is complete.

\section{Some Optimal Tomographic Goalies}

The tomographic goalie problem is a primal LP problem, as outlined below:

$$
\begin{aligned}
& \text { Minimize } \int f(x) d x \\
& \text { subject to } f(x) \geq 0 \text { and } \int_{\ell(\theta, p)} f(x) \geq \omega_{C}(\ell(\theta, p)),
\end{aligned}
$$

where $\omega_{C}(\ell)$ is equal to $1 / \operatorname{diam}(C, \ell)$ if $\ell$ hits $C$ and is equal to 0 if $\ell$ misses $C$. The tomographic in-goalie problem is identical except that we impose the additional condition $f(x)=0$ for $x \notin C$. Points $x$ where we may have $f(x)>0$ are termed admissible.

The dual to the tomographic goalie problem can be formulated as follows. The variables are indexed by lines in the plane. We therefore consider densities on lines $g(\ell)$. The densities are constrained to be non-negative $g(\ell) \geq 0$ and they satisfy the following back-projection condition on (almost all) admissible $x$ :

$$
\int_{0}^{\pi} g\left(\ell\left(\theta, p_{x}(\theta)\right)\right) d \theta \leq 1
$$


where $p_{x}(\theta)=\langle x, \vec{\theta}\rangle$ and $p$ is such that $x \in \ell(\theta, p)$. The dual objective is to maximize the following quantity:

$$
\iint g(\ell(\theta, p)) \omega_{C}(\ell(\theta, p)) d p d \theta
$$

LP duality provides the following inequality.

$$
\begin{aligned}
\iint g( & (\theta, p)) \omega_{C}(\ell(\theta, p)) d p d \theta \\
& \leq \iint g(\ell(\theta, p))\left(\int \delta\left(p_{x}(\theta)-p\right) f(x) d x\right) d p d \theta \\
& \leq \int g\left(\ell\left(\theta, p_{x}(\theta)\right)\right)\left(\int f(x) d x\right) d \theta \\
& \leq \int f(x) d x .
\end{aligned}
$$

Thus, we can prove optimality by finding primal-dual pairs. We have already presented the optimal tomographic goalie for the disk, which happens to be an in-goalie, we will now do the same for the triangle and the square.

\subsection{The Triangle}

The optimal tomographic in-goalie for the equilateral triangle is indicated in Fig. 7 along with its dual measure. The density $f$ is a uniform density on the boundary of the triangle with total mass $\frac{3}{2}$. The proof of feasibility of $f$ can be found in the Appendix. The optimal dual density $g$ is supported by the three directions parallel to the sides. Each direction carries total mass $\frac{1}{2}$ uniformly distributed over the lines that intersect the triangle between the center and the vertex opposite the side parallel to the given direction. For any point in the triangle, at most one direction has non-zero back projection. Therefore, $g$ is feasible and since the total mass of $g$ is $\frac{3}{2}$ we conclude that the presented solution is optimal.

There is an optimal IP triangle goalie that blocks the directions supported by the dual measure, see Fig. 8.

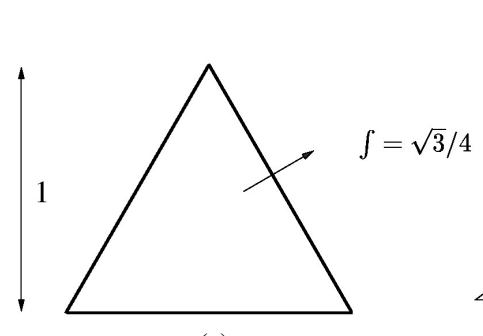

(a)

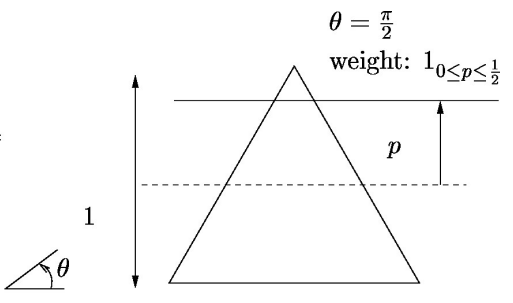

(b)

Fig. 7. Optimal tomographic in-goalie for the triangle. Primal and dual optimal solutions are indicated. (a) Primal solution-uniform density on boundary. (b) Dual density function in direction parallel to bottom face: 1 on upper half of triangle. 


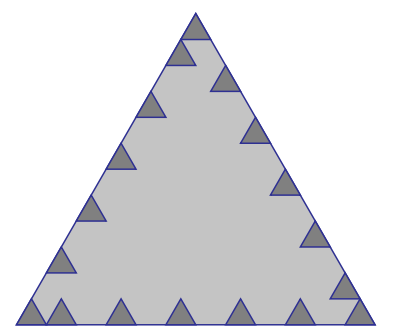

Fig. 8. Six direction IP goalie for the triangle.

\subsection{The Square}

The optimal tomographic in-goalie for the square is indicated in Fig. 9 along with the dual measure used to prove optimality. The density $f$ is the sum of a uniform density concentrated on the boundary of the square of total mass $\frac{2}{3}$ and another uniform density concentrated on the two diagonals also of total mass $\frac{2}{3}$ giving total mass $\frac{4}{3}$. The proof of feasibility of $f$ can be found in the Appendix. Bárány and Füredi [BF] proved the lower bound $\frac{4}{3}$. The dual density $g$ is supported by four directions: those parallel to the sides and those parallel to the diagonals.

The four-direction LP goalie of Kern and Wanka is similar to the tomographic goalie presented here. Their solution fails to be a goalie for all directions but it is clear how to improve it in view of the tomographic goalie. The weakness in Kern and Wanka's solution can be seen as arising simply from quantizing the tomographic goalie too coarsely. They considered goalies for $n S$ using only unit squares translated by integers. We do not impose this constraint in general but, even under this constraint, we can construct an LP goalie based on the tomographic goalie that blocks all directions using total weight $\frac{4}{3} n+O(\sqrt{n})$. Figure 10 presents an example of the construction which is evaluated below.

Consider the unit square $S$ and consider $n S$ tiled with translates of $S$. We will now assign weights to the integer translates of $S$ in $n S$ to construct an LP goalie.

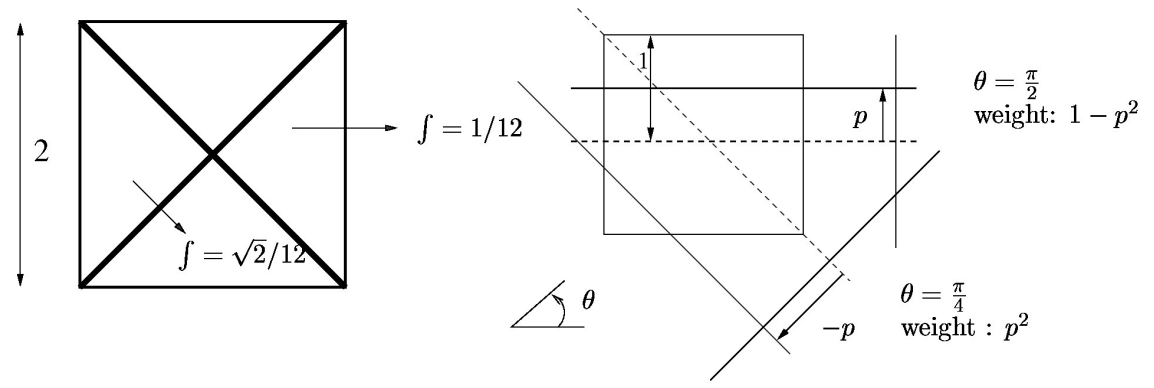

(a)

(b)

Fig. 9. Optimal tomographic in-goalie for the square. The value of integrating perpendicular to the uniform densities is indicated. (a) Primal solution-sum of uniform density on boundary and on diagonals. (b) Dual density function in two of four directions. 


\begin{tabular}{|c|c|c|c|c|c|c|c|c|c|c|c|c|c|c|c|c|c|c|c|c|c|c|c|}
\hline & $a$ & a & a & a & a & $\mathrm{a}$ & a & $\mathrm{a}$ & a & a & $\mathrm{a}$ & a & $\mathrm{a}$ & $\mathrm{a}$ & $\mathrm{a}$ & a & $a$ & $\mathrm{a}$ & a & $\mathrm{a}$ & $\mathrm{a}$ & a & \\
\hline$a$ & & $a$ & $a$ & $a$ & $a$ & $a$ & a & $a$ & $a$ & $a$ & $a$ & $a$ & $a$ & $a$ & $a$ & $a$ & $a$ & $a$ & $\mathrm{a}$ & $a$ & $a$ & & \\
\hline d & $a$ & & $a$ & a & $c$ & $a$ & $\mathrm{a}$ & $\mathrm{a}$ & $\mathrm{a}$ & $\mathrm{a}$ & a & a & $a$ & $\mathrm{a}$ & $a$ & a & $a$ & $a$ & $\mathrm{a}$ & $a$ & & a & \\
\hline a & a & a & & a & $a$ & $a$ & $\mathrm{a}$ & a & a & $a$ & a & a & $a$ & $\bar{a}$ & $a$ & $a$ & a & $\mathrm{a}$ & a & & a & $\mathrm{a}$ & \\
\hline$a$ & a & $\mathrm{a}$ & $a$ & $1 / 3$ & & & & & & & & & & & & & & & $1 / 3$ & $\mathrm{a}$ & $a$ & $a$ & a \\
\hline $\mathrm{a}$ & $a$ & a & a & & $1 / 3$ & & & & & & & & & & & & & $1 / 3$ & & $\mathrm{a}$ & $a$ & $a$ & \\
\hline a & $a$ & a & $a$ & & & $1 / 3$ & & & & & & & & & & & & & & $\mathrm{a}$ & $\mathrm{a}$ & $a$ & $\bar{a}$ \\
\hline $\mathrm{a}$ & a & a & $a$ & & & & $1 / 3$ & & & & & & & & & $1 / 3$ & & & & $a$ & $a$ & $a$ & a \\
\hline a & $a$ & $a$ & $\mathrm{a}$ & & & & & $1 / 3$ & & & & & & & $1 / 3$ & & & & & $\mathrm{a}$ & $\mathrm{a}$ & $\mathrm{a}$ & a \\
\hline $\mathrm{a}$ & $a$ & $a$ & $a$ & & & & & & $1 / 3$ & & & & & $1 / 3$ & & & & & & $a$ & $a$ & $a$ & $a$ \\
\hline$a$ & $a$ & $a$ & a & & & & & & & $1 / 3$ & & & $1 / 3$ & & & & & & & $\mathrm{a}$ & $a$ & $a$ & a \\
\hline$a$ & $a$ & $a$ & $a$ & & & & & & & & $1 / 3$ & $1 / 3$ & & & & & & & & $\mathrm{a}$ & $a$ & $a$ & $a$ \\
\hline $\mathrm{a}$ & a & a & a & & & & & & & & $1 / 3$ & $1 / 3$ & & & & & & & & $\mathrm{a}$ & $\mathrm{a}$ & $a$ & a \\
\hline$a$ & a & $a$ & $\mathrm{a}$ & & & & & & & $1 / 3$ & & & $1 / 3$ & & & & & & & a & $\mathrm{a}$ & $\mathrm{a}$ & $a$ \\
\hline$a$ & $a$ & $a$ & $a$ & & & & & & $1 / 3$ & & & & & $1 / 3$ & & & & & & $a$ & $a$ & $a$ & $a$ \\
\hline$a$ & $a$ & $a$ & a & & & & & $1 / 3$ & & & & & & & $1 / 3$ & & & & & $\mathrm{a}$ & $\mathrm{a}$ & $a$ & $\mathrm{a}$ \\
\hline $\mathrm{a}$ & $a$ & $\mathrm{a}$ & $a$ & & & & $1 /$ & & & & & & & & & $1 / 3$ & & & & $\mathrm{a}$ & $a$ & $a$ & $a$ \\
\hline$a$ & $\mathrm{a}$ & $\mathrm{a}$ & a & & & $1 / 3$ & & & & & & & & & & & $1 / 3$ & & & $\mathrm{a}$ & $\mathrm{a}$ & $a$ & $a$ \\
\hline $\mathrm{a}$ & a & $\mathrm{a}$ & $\mathrm{a}$ & & $1 / 3$ & & & & & & & & & & & & & $1 / 3$ & & $\mathrm{a}$ & $a$ & $\mathrm{a}$ & $\mathrm{a}$ \\
\hline $\mathrm{a}$ & $\mathrm{a}$ & $\mathrm{a}$ & $a$ & 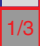 & & & & & & & & & & & & & & & $1 / 3$ & $\mathrm{a}$ & $\mathrm{a}$ & a & $a$ \\
\hline$a$ & $a$ & $\mathrm{a}$ & & $a$ & $a$ & $\mathrm{a}$ & a & $a$ & $a$ & $a$ & $a$ & $a$ & $a$ & $a$ & $\mathrm{a}$ & $\mathrm{a}$ & $a$ & $\mathrm{a}$ & $a$ & & $\bar{a}$ & $a$ & a \\
\hline a & $a$ & & $a$ & $a$ & $\mathrm{a}$ & $\mathrm{a}$ & $a$ & $a$ & $\mathrm{a}$ & $a$ & $a$ & $\mathrm{a}$ & $a$ & $a$ & $\mathrm{a}$ & $\mathrm{a}$ & $a$ & $a$ & $a$ & $a$ & & $a$ & $a$ \\
\hline$a$ & & a & $a$ & $a$ & $\mathrm{a}$ & $\mathrm{a}$ & $a$ & $\mathrm{a}$ & a & $a$ & $a$ & $a$ & $a$ & $a$ & $a$ & $\mathrm{a}$ & $a$ & $\mathrm{a}$ & $\mathrm{a}$ & $\mathrm{a}$ & $a$ & & \\
\hline & $\mathrm{a}$ & $a$ & $a$ & $a$ & 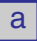 & $a$ & 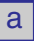 & $a$ & $a$ & $a$ & $a$ & $a$ & $a$ & $a$ & $\mathrm{a}$ & $a$ & $a$ & $\mathrm{a}$ & $\mathrm{a}$ & $a$ & a & a & \\
\hline
\end{tabular}

Fig. 10. LP goalie construction, $k=4, a=(4 k /(4 k-1))(1 / 6 k)$.

Enumerate the rows and columns $1, \ldots, n$. Choose a parameter $k<n / 2$. For $k<$ $i, j \leq n-k$, set the weight $w_{i, j}=\frac{1}{3}\left(\delta_{i-j}+\delta_{i+j-n-1}\right)$. Otherwise set the weight $w_{i, j}=\max \left\{\delta_{i-j}+\delta_{i+j-n-1},(4 k /(4 k-1))(1 / 6 k)\right\}$. This defines the weights, see Fig. 10.

Now, consider the infinite tiling. Consider putting positive weight on all squares in a horizontal infinite strip of width $k$ and on all squares in a vertical infinite strip of width $k$. Furthermore, on the intersection of the strips assign the sum of the weights, i.e., 2. We claim that any line in the plane which is not either vertical or horizontal accumulates total weight at least $4 k-1$.

To prove the claim consider any line crossing both strips. Let $h$ be the vertical height between the intersection points on the boundary of the vertical strip. Without loss of generality we may assume $h \geq k$. It is not hard to see that the total weight accumulated by the line from the vertical strip is at least $k+\lfloor h\rfloor-1$ and the weight accumulated by the line from the horizontal strip is at least $k+\left\lfloor k^{2} / h\right\rfloor-1$. Thus, the total accumulated weight is at least $2 k-2+\lfloor h\rfloor+\left\lfloor k^{2} / h\right\rfloor$. It is not hard to show that this quantity is minimized for $h$ just slightly larger than $k$ where it takes the value $4 k-1$.

Using the above result it is easy to verify that the proposed weights do indeed yield a goalie. The total weight of the goalie is $2 \frac{1}{3}(n-2 k)+4 k+4(4 k /(4 k-1))(1 / 6 k)(k(n-$ $\left.2 k)+k^{2}-k\right)$. If we set $k=\lfloor\sqrt{n}\rfloor$, then the total weight is easily seen to be $\frac{4}{3} n+O(\sqrt{n})$. 


\section{Acknowledgments}

We thank Imre Bárány for the significant improvement of the paper that resulted from his comments on earlier versions of it. We also thank Jim Reeds for stimulating discussions and for suggesting the name "goalie", which comes from the theory of games (like soccer and hockey), where the ball or puck is assumed to travel along straight lines.

\section{Appendix. Feasibility of Tomographic In-Goalies}

Here we prove the feasibility of the tomographic goalies presented in Section 4.

The Triangle. We will show that the indicated primal density on the triangle is feasible. Let $\ell$ be a line making an angle $\varphi$ with the bottom face of the triangle which intersects the other two faces. See Fig. 11. We will show $\operatorname{diam}(C, \ell) \int_{\ell} f \geq 1$ where $C$ is the triangle of unit height. By symmetry we need only consider $0 \leq \varphi \leq \pi / 3$. We have

$$
\int_{\ell} f=\frac{\sqrt{3}}{4}\left(\frac{1}{\cos (\pi / 6+\varphi)}+\frac{1}{\cos (\pi / 6-\varphi)}\right)
$$

and

$$
\operatorname{diam}(C, \ell)=\frac{2}{\sqrt{3}} \cos \left(\frac{\pi}{6}-\varphi\right) .
$$

The inequality to be shown, $(\operatorname{diam}(C, \ell)) \int_{\ell} f \geq 1$, is therefore equivalent to $\cos (\pi / 6$ $-\varphi) \geq \cos (\pi / 6+\varphi)$ which holds for $0 \leq \varphi \leq \pi / 3$.

The Square. We will now show that the indicated primal density on the square is feasible. Let $\ell$ be a line making an angle $\varphi$ with the bottom face of the square which intersects the square but not the bottom face. Thus, we have $-\pi / 2<\varphi<\pi / 2$. We will show $\operatorname{diam}(C, \ell) \int_{\ell} f \geq 1$ where $C$ is the square of height 2 . By symmetry we need only consider $0 \leq \varphi \leq \pi / 2$. There are two cases depending on whether $\ell$ hits the two parallel sides of the square or one side and the top, see Fig. 12.

Case 1: $\ell$ hits parallel sides. We have

$$
\int_{\ell} f=2 \frac{1}{12} \frac{1}{\cos (\varphi)}+\frac{\sqrt{2}}{12}\left(\frac{1}{\cos (\pi / 4-\varphi)}+\frac{1}{\cos (\pi / 4+\varphi)}\right)
$$

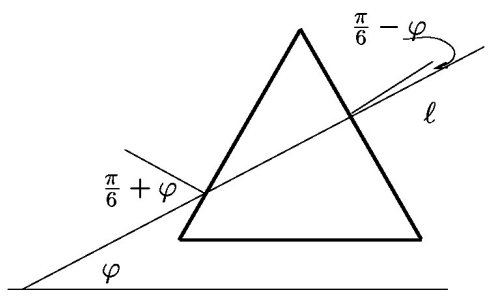

Fig. 11. Feasibility of tomographic goalie for the triangle. 


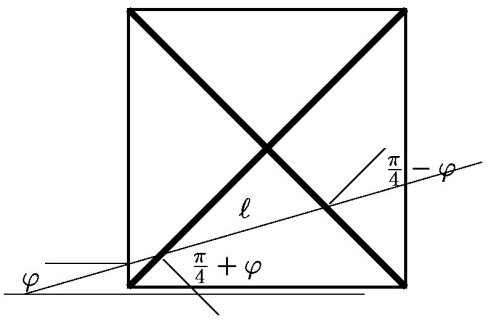

Case 1.

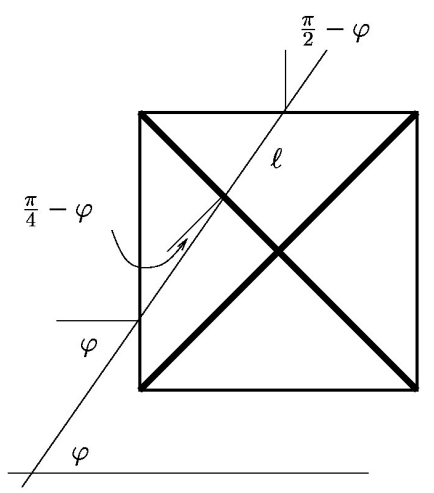

Case 2.

Fig. 12. Feasibility of tomographic goalie for the square.

and

$$
\operatorname{diam}(C, \ell)=2 \sqrt{2} \cos \left(\frac{\pi}{4}-\varphi\right),
$$

while $0 \leq \varphi \leq \pi / 4$. Thus, the inequality to be shown, $\operatorname{diam}(C, \ell) \int_{\ell} f \geq 1$, is equivalent to

$$
\frac{\sqrt{2}}{3} \frac{\cos (\pi / 4-\varphi)}{\cos \varphi}+\frac{1}{3}\left(1+\frac{\cos (\pi / 4-\varphi)}{\cos (\pi / 4+\varphi)}\right) \geq 1 .
$$

The left side of this expression is increasing in $\varphi$ for $\varphi \in[0, \pi / 4]$ and, since equality holds at $\varphi=0$, the inequality is satisfied in the entire interval.

Case 2: $\ell$ hits left side and top. We have

$$
\int_{\ell} f=\frac{1}{12}\left(\frac{1}{\cos (\varphi)}+\frac{1}{\cos (\pi / 2-\varphi)}\right)+\frac{\sqrt{2}}{12} \frac{1}{\cos (\pi / 4-\varphi)}
$$

and

$$
\operatorname{diam}(C, \ell)=2 \sqrt{2} \cos \left(\frac{\pi}{4}-\varphi\right),
$$

while $\pi / 4 \leq \varphi \leq \pi / 2$. Here, the inequality $\operatorname{diam}(C, \ell) \int_{\ell} f \geq 1$ is seen, after some manipulation, to be equivalent to $\frac{1}{6}(2+\tan (\varphi)+\cot (\varphi))+\frac{1}{3} \geq 1$, which holds for $\pi / 4 \leq \varphi \leq \pi / 2$.

\section{References}

[BF] I. Bárány, and Z. Füredi, Covering all secants of a square, in Intuitive Geometry (G. Fejes Tóth, ed.), Colloq. Math. Soc. János Bolyai, vol. 48 (Siófok, Hungary, 1985), pp. 19-27, North-Holland, Amsterdam, 1987. Also available as Rutcor Research Report 3-86, March 1986.

[Br] K. A. Brakke, The opaque cube problem, Amer. Math. Monthly 93 (1986), 866-871.

[Cr] H. T. Croft, Curves intersecting certain sets of great circles on the sphere, J. London Math. Soc. (2) 1 (1969), 461-469. 
[Eg] H. G. Eggleston, The maximal in-radius of the convex cover of a plane connected set of given length, Proc. London Math. Soc. (3) 45 (1982), 456-478.

[EP] P. Ërdos and J. Pach, On a problem of L. Fejes Tóth, Discrete Math. 30(2) (1980), 103-109.

[FM] V. Faber and J. Mycielski, The shortest curve that meets all the lines that meet a convex body. Amer. Math. Monthly 93 (1986), 796-801.

[FMP] V. Faber, J. Mycielski, and P. Pedersen, On the shortest curve that meets all the lines which meet a circle, Ann. Polon. Math. 44 (1984), 249-266.

[KW] W. Kern and A. Wanka, On a problem about covering lines by squares, Discrete Comput. Geom. 5 (1990), 77-82.

[Ma] E. Makai, Jr., On a dual of Tarski's plank problem, Diskrete Geometrie, 2, Kolloq., Inst. Math. Univ. Salzburg, 1980, pp. 127-132.

[MS] T. Marzetta and L. Shepp, A surprising Radon transform result, and its application to motion detection, Preprint.

[Sa] L. A. Santaló, Integral Geometry and Geometric Probability, Addison-Wesley, Reading, Massachusetts, 1976.

[To] L. Fejes Tóth, Remarks on dual of Tarski's plank problem, Mat. Lapok 25 (1974), 13-20.

[Va] P. Valtr, Unit squares intersecting all secants of a square, Discrete Comput. Geom. 5 (1990), 77-82.

Received August 24, 1999, and in revised form December 31, 2002, April 21, 2003, and May 6, 2003.

Online publication October 3, 2003. 